\title{
The long-term ingestion of a diet high in extra virgin olive oil produces obesity and insulin resistance but protects endothelial function in rats: a preliminary study
}

\author{
Hady Keita ${ }^{1}$, Eduardo Ramírez-San Juan', Norma Paniagua-Castro ${ }^{1}$, Leticia Garduño-Siciliano ${ }^{2}$ and Lucía Quevedo ${ }^{1 *}$
}

\begin{abstract}
Background: It has been hypothesized that fatty acids derived from a diet high in saturated fat may negatively affect endothelial function more significantly than a diet high in unsaturated fat; nevertheless, the effects of the long-term ingestion of monounsaturated fatty acids on endothelial function have been poorly studied.

Methods: To examine the chronic effects of monounsaturated (e.g., extra virgin olive oil (EVOO)) or saturated (e.g., margarine (M)) fatty acid-rich diets on the development of insulin resistance and endothelial dysfunction in rats, three groups of rats were fed control, high-EVOO or high-M diets for 20 weeks. Body weight, energy consumption, insulin resistance, lipid peroxidation and in vitro vascular reactivity with and without metformin were assessed during the study period.

Results: Both high-fat diets produced obesity and insulin resistance. EVOO-fed rats showed smaller increases in total cholesterol and arterial lipid peroxidation when compared with M-fed rats. Vascular reactivity to phenylephrine and sodium nitroprusside was not modified, but the vasodilating effect of carbachol was especially reduced in the M-fed rats compared with the EVOO-fed or control groups. Metformin addition to the incubation media decreased the vascular response to phenylephrine; decrease that was lower in rats fed with both high fat diets, and increased the carbachol and nitroprusside effects, but the metformin-enhanced response to carbachol was lower in the M group.

Conclusions: Our results suggest that feeding rats with high quantities of EVOO, despite producing obesity and insulin resistance, produces low levels of circulating cholesterol and arterial lipoperoxidation compared to $\mathrm{M}$ fed rats and shows a preserved endothelial response to carbachol, effect that is significantly enhanced by metformin only in rats fed with control and EVOO diets.
\end{abstract}

Keywords: Endothelial dysfunction, Obesity, Extra virgin olive oil, Insulin resistance

\section{Background}

During the last few decades, sedentary lifestyles and transient changes in consumption patterns, including a sharp increase in fat consumption from vegetable oils and animal-based food sources, have led to drastic increases in the prevalence of overweight and obese individuals in many developed and developing countries [1]. The

\footnotetext{
* Correspondence: quevedocorona@hotmail.com

'Departamento de Fisiología, Escuela Nacional de Ciencias Biológicas, Instituto Politécnico Nacional, Carpio y Plan de Ayala, México, D.F., México Full list of author information is available at the end of the article
}

importance of fat consumption in the development of insulin resistance, type 2 diabetes mellitus and cardiovascular disease has also been shown [2,3], but differences in weight gain and predisposition to cardiovascular disease in some populations suggest that dietary fat composition plays an important role in determining the development of these chronic diseases [4,5]. Diets rich in saturated fatty acids (SAFA) have been linked to endothelial dysfunction, which contributes to macrovascular alterations. On the other hand, the Mediterranean diet, which is mainly characterized by abundant vegetables, fruits, 
complex carbohydrates, large amounts of olive oil as the main dietary fat, low fat dairy and animal products which guarantees the low consumption of saturated fats, has been recognized for its positive effects on cardiovascular health [6,7] and its protective effect against the development of obesity and diabetes [8-10]. In the Mediterranean region, total daily lipid intake may contribute with the $40 \%$ of total energy intake in Greece, or 30\% in Italy, hence, olive oil plays a central role in this diet [11]. Many studies have attributed some of the beneficial effects of the Mediterranean diet to olive oil phenolic compounds with high antioxidant capacity, e.g., hydroxytyrosol, tyrosol, oleuropein aglycon and its derivatives, which are naturally found in extra virgin olive oil and to the high proportion of monounsaturated fatty acids (MUFA), namely oleic acid [12-14]. EVOO polyphenols have been reported having strong anti-inflammatory properties [14], decrease oxidized low-density lipoproteins, decrease blood pressure and improve endothelial function in humans [15] and prevent oxidative DNA damage [16]. Interestingly, in a recent study, an extra virgin olive oil, rich in oleic acid, has been shown having antioxidant properties and an improved adaptive response of the body in rats submitted to exhaustive exercise [17].

It has also been reported that MUFA consumption leads to a decrease in low-density lipoprotein (LDL) cholesterol [18], while saturated fat intake increases LDL levels $[19,20]$. Concurrently, other studies have suggested that unsaturated fat increases LDL cholesterol in a manner similar to what has been observed with saturated fat, but saturated fat, especially trans fats, simultaneously decreases high-density lipoprotein (HDL) cholesterol levels and increase the deposition of cholesterol into cellular plasma membranes in vascular tissues, leading to the development of atherosclerosis over time [21,22]. The general consensus is that the dietary intake of SAFA increases cardiovascular risk but that MUFA and polyunsaturated fatty acids (PUFA) intake decreases risk [23]. Contrarily, it has been shown that increased circulating concentrations of cholesterol and free fatty acids reduce the endothelial bioavailability of nitric oxide (NO) due to both the increased generation of superoxide and a reduction in endothelial NO synthase activity [24,25]. Reactive oxygen species can react with the cell membrane PUFAs, causing lipid peroxidation and thus altering the vasodilatory function of the endothelium [26]. Furthermore, adipose tissue secretes free fatty acids and cytokines, a process that is intimately involved in the development of insulin resistance and contributes to a proinflammatory state which, overall, puts a patient at an increased cardiovascular risk [27-29]. Many studies have shown that vascular complications are a major cause of mortality in diabetic patients [30] and that the endothelium plays a critical role in controlling vascular tone and blood flow by synthesizing mediators of vasodilation and vasoconstriction.

Impaired endothelium-dependent vasodilation in obesityassociated insulin resistance [31] and diabetes [32] is characterized by a diminished NO production and the development of a pro-inflammatory vascular phenotype that promotes atherosclerosis and adverse cardiovascular events. Previous studies of rats fed with high-fat diets enriched with olive oil for 4 weeks showed that the rats achieved low body weights [33] and retained a normal insulin sensitivity [34]. In contrast, Buettner et al. [35] found that feeding rats a diet high in EVOO over a long period of time produces obesity and insulin resistance, similar to what is seen in animals fed a lard-based diet. These findings suggest that obesity and the deleterious effects on vascular integrity of ingesting high quantities of fats may only occur with chronic ingestion. Most of the recent studies on beneficial effects of EVOO were conducted with either well balanced or hypocaloric diets, but there are scant and contradictory data about the effects due to a high EVOO ingestion, therefore the aim of this study was to determine if the long-term consumption of a hypercaloric diet high in monounsaturated fatty acids such as the extra virgin olive oil produces obesity, insulin resistance and endothelial dysfunction in rats as well as compare these effects with those of a hypercaloric diet high in saturated fat.

\section{Methods}

\section{Animals and diets}

Twenty seven male Wistar rats, obtained from the University of Hidalgo and initially weighing between 120-150 g (5-6 wk old), were housed in individual cages in a temperature-regulated room $\left(22^{\circ} \mathrm{C} \pm 2^{\circ} \mathrm{C}\right)$ with 12-hour dark-light cycles (lights on from 7:00 AM to 7:00 PM). After one week of acclimatization, nine rats were assigned to each diet group (control, EVOO, M), and the specialized feedings were initiated. The animals had free access to their respective diets. The control animals were fed a commercial diet (Rodent Laboratory Diet 5001 PMI, Richmond, IN), with a total energy content of $13.68 \mathrm{~kJ} / \mathrm{g}$. The remaining animals received a high-fat diet (25\% extra virgin olive oil or margarine was added to the standard diet). Both high-fat diets had an energy content of $19.68 \mathrm{~kJ} / \mathrm{g}$ (54.2\% of total energy derived from fat). We used an Italian EVOO containing $474.2 \mathrm{mg} / \mathrm{kg}$ polyphenols that was purchased in a local market. The total phenol content was determined colorimetrically at $725 \mathrm{~nm}$ following the Folin \& Ciocalteau method in the extracts in triplicate as previously described [36] and expressed as milligrams of galic acid equivalents per kilogram of oil. The EVOO comprised $15.44 \%$ saturated fat, $74.47 \%$ unsaturated fat and $10.07 \%$ polyunsaturated fat; while, the $M$ comprised $57.14 \%$ saturated fat, $25.71 \%$ unsaturated fat and $17.14 \%$ polyunsaturated. The macronutrient compositions 
of the diets are shown in Table 1. The food consumption per animal was measured daily, and body weights were determined for each animal once a week over the 20-week period. All of the experimental procedures were conducted according to the recommendations of the Guide for the Care and Use of Laboratory Animals of the Mexican Council for Animal Care (NOM-062-ZOO-1999) and were approved by the National School of Biological Sciences Ethics and Biosecurity Committee.

\section{Reagents}

(R)-(-)-phenylephrine hydrochloride, isoproterenol hydrochloride, sodium nitroprusside and metformin (1.1 dimethylbiguanide hydrochloride) were all purchased from Sigma and Sigma-Aldrich, and carbachol was purchased from Research Biochemicals International. Rat/mouse insulin ELISA kits were purchased from Millipore (Linco Research Inc., St. Charles, MO, USA).

\section{Blood and serum measurements}

After twenty weeks of experimental feeding, blood samples were obtained from the tips of the rats' tails at 8:00 AM, following an overnight 12-hour fast. The glucose levels were determined in whole blood with a glucose meter (Optium medisense, Abbott Laboratories, Oxford, United Kingdom). The samples $(0.5 \mathrm{~mL})$ were collected at the conclusion of the experiment to determine the total cholesterol, HDL-cholesterol and triglyceride (TG) levels using specific enzymatic-colorimetric kits (Randox, Crumlin, UK). The samples were centrifuged at $3500 \mathrm{rpm}$ for $15 \mathrm{~min}$ at $4^{\circ} \mathrm{C}$. Plasma LDL- cholesterol levels were calculated using the formula of Friedeward et al. [37].

\section{Blood glucose and serum insulin levels during the} intraperitoneal glucose tolerance test

An intraperitoneal (IP) glucose tolerance test (GTT) was performed in the rats after an overnight fast of 12 hours.

Table 1 Macronutrient composition of study diets (g/100 g)

\begin{tabular}{llll}
\hline $\begin{array}{l}\text { Component } \\
\text { g/100 g }\end{array}$ & $\begin{array}{l}\text { Control } \\
\text { diet }\end{array}$ & $\begin{array}{l}\text { EVOO } \\
\text { High-fat diet }\end{array}$ & $\begin{array}{l}\text { Margarine } \\
\text { High-fat diet }\end{array}$ \\
\hline Protein & 23.0 & 17.25 & 17.25 \\
Carbohydrate & 48.7 & 36.53 & 36.53 \\
Total fat & 4.5 & 28.37 & 28.37 \\
Fat from chow & 4.5 & 3.37 & 3.37 \\
EVOO & - & 25.0 & - \\
Margarine & - & - & 25.0 \\
Fiber & 6.0 & 4.5 & 4.5 \\
Minerals & 7.0 & 5.3 & 5.3 \\
Vitamins & 1.0 & 0.75 & 0.75 \\
Moisture & 10.0 & 7.5 & 7.5 \\
\hline
\end{tabular}

EVOO extra virgin olive oil.
Basal blood samples were obtained from the tip of the tail, and a $35 \%$ glucose solution was intraperitoneally injected (3 g/kg body weight). At 15, 30, 60, 120 and 180 minutes post-glucose administration, blood glucose levels were determined [38]. Fasting blood samples $(0.25 \mathrm{~mL})$ were obtained in basal conditions and were centrifuged at $3500 \mathrm{rpm}$ for 15 minutes at $4^{\circ} \mathrm{C}$. The resulting serum was kept frozen at $-70^{\circ} \mathrm{C}$ until insulin levels were determined [39]. Insulin resistance was evaluated using the homeostasis model assessment of insulin resistance (HOMA-IR), which is calculated as the product of the fasting serum insulin $(\mu \mathrm{U} / \mathrm{mL})$ and the fasting blood glucose (mg/dL) divided by 2430 [40].

\section{Intraabdominal fat pad weights}

At week 20, the rats were anesthetized with diethyl ether and euthanized by decapitation. The thoracic region was immediately dissected, and a segment of aorta, between the arch and the diaphragm, was removed for use in the study of endothelial function. The abdominal region was then dissected, and the intraabdominal fat deposits were excised and weighed. Accurately weighed samples from the aorta, liver and heart were also taken for the lipoperoxidation assay.

\section{Lipid peroxidation assay}

Lipid peroxidation in the artery, heart and liver was assessed by measuring the malondialdehyde (MDA) content, as described by Buege and Aust [41], but with minor modifications. Briefly, $50 \mathrm{mg}$ of tissue was homogenized in $0.5 \mathrm{~mL}$ of cold phosphate buffer ( $\mathrm{pH} 7.4)$, followed immediately with the addition of $1 \mathrm{~mL}$ of a reagent containing trichloroacetic acid, thiobarbituric acid and $\mathrm{HCl}[15 \%(\mathrm{w} / \mathrm{v}), 0.375 \%(\mathrm{w} / \mathrm{v})$ and $0.25 \mathrm{~N}$, respectively] to the homogenate. This solution was then heated for $60 \mathrm{~min}$ in a boiling water bath. After cooling, the flocculent precipitate was removed by centrifugation at $1000 \mathrm{~g}$ for $10 \mathrm{~min}$. A spectrophotometer was used to determine the absorbance at $532 \mathrm{~nm}$. A sample containing reagent, only, was used as a blank. The MDA content was calculated using an extinction coefficient of $1.56 \times$ $10^{-5} \mathrm{M}^{-1} \mathrm{~cm}^{-1}$. The protein content was determined using the Coomassie blue method [42], with bovine serum albumin as the standard.

\section{Measurement of isometric force}

The segment of thoracic aorta was placed in oxygenated, modified Krebs-Hanseleit solution (KHS) $(\mathrm{NaCl}, \mathrm{KCl}$, $\mathrm{NaHCO}_{3}, \mathrm{CaCl}_{2}, \mathrm{NaHPO}_{4}, \mathrm{MgSO}_{4}$, dextrose and EDTA). The thoracic aorta was cleaned of loosely adhering fat and connective tissue. It was cut into 3-mm-wide rings and then placed in an oxygenated $\left(95 \% \mathrm{O}_{2}, 5 \% \mathrm{CO}_{2}\right)$ bath with $10 \mathrm{ml}$ of $\mathrm{KHS}$, with one end of the specimen connected to a tissue holder and the other to a 
transducer (TSD125C-50 g, BIOPAC Systems Inc. Santa Barbara California, Model MP100). First, the aortic ring was equilibrated for 45 min under a resting tension of 2.0 g. After equilibration, the aortic rings were precontracted with phenylephrine $\left(1 \times 10^{-6} \mathrm{M}\right)$, and after reaching a stable plateau phase were exposed to carbachol $\left(1 \times 10^{-6} \mathrm{M}\right)$ to verify the functionality of endothelium. Contraction was evoked again with phenylephrine $\left(1 \times 10^{-6} \mathrm{M}\right)$, then cumulative concentration-response curves to sodium nitroprusside or carbachol were determined from $1 \times 10^{-8}$ to $1 \times 10^{-4} \mathrm{M}$ for the relaxation studies. In another set of experiments, cumulative concentration-response curves to phenylephrine $\left(1 \times 10^{-8}\right.$ to $1 \times 10^{-4} \mathrm{M}$ ) were generated.

Metformin has been widely used to increase glucose and lipid metabolism in patients and rodent models of type 2 diabetes mellitus and insulin resistance to improve glycemic control and lower blood pressure. However, the insulin resistance mechanisms to increase incidence of vascular disease are not completely understood [43]. Additionally, as metformin has been reported to relax isolated arterial preparations through a nitric oxide dependent mechanism [43], we hypothesized that addition of metformin to the incubation media should improve the response to vascular active substances, showing differential effects in relation to fat diet composition. Thus, after performing the previous experiments, the aortic ring segments were washed sufficiently to recover their basal contraction $(2.0 \mathrm{~g})$ and were incubated in metformin $\left(10^{-4} \mathrm{M}\right)$ for $40 \mathrm{~min}$, finally, the first experiment was repeated using the same incubation medium.

\section{Statistical analyses}

The results are presented as the means \pm SEM, and oneway ANOVA, two-way RM-ANOVA or three-way RMANOVA analyses were used to compare the mean values among the different groups, followed by further analysis with the Student-Newman-Keuls' post hoc test. In all cases, a P value of 0.05 or less (two-tailed) was considered statistically significant.

\section{Results}

\section{Food consumption, energy intake and body weight}

The rats fed with extra virgin olive oil and margarine diets ingested significantly less food than the control diet rats $\mathrm{P}<0.05$ (data not shown); however, the overall energy intake and body weights were significantly higher in both of the high-fat diet group rats (EVOO and $\mathrm{M}$ ) than in the control group $(\mathrm{P}<0.05$; Figures $1 \mathrm{~A}$ and $1 \mathrm{~B})$ at the conclusion of the 20 weeks of dietary treatment. No significant differences in these variables between the two high-fat diets were found.

\section{Glycemia and glucose tolerance}

At week 20 of dietary treatment, an IP injection of a glucose solution $(3 \mathrm{~g} / \mathrm{kg})$ produced a significant increase in glycemia after 15 minutes in all animals. At 30 minutes, blood glucose levels decreased only in the control rats and were significantly lower in these rats compared with the HF-fed rats. At 180 minutes, the control diet group had recovered to their baseline blood glucose levels, but the HF-diet groups had not (Figure 2A). The area under glucose curve was significantly greater in the HF-diet groups than in the control group (Figure 2B). However, the corresponding insulin levels under fasting conditions were not different between the groups. The HOMA-IR analysis revealed significant differences in insulin resistance between the HF-diet groups and the control group (Table 2).

Serum total cholesterol, lipoprotein cholesterol (HDL and LDL) and triglycerides (TG)

Total cholesterol, HDL, LDL and TG plasma concentrations after 20 weeks of dietary treatment varied between the experimental groups. M-fed rats showed higher total and LDL-cholesterol plasma levels than those found in the control or EVOO-fed rats $(\mathrm{P}<0.05)$, but HDL and TG levels did not differ significantly between the dietary groups (Table 3).

\section{Intraabdominal fat pad weights}

The combined weight of the retroperitoneal, epididymal and mesenteric fat pads, expressed as a percentage of body weight, was greater for the HF-diets rats than for the control rats (Table 3).

\section{Lipid peroxidation}

Malondialdehyde levels in the thoracic aortas, hearts and livers of the M-diet group were significantly higher than the levels found in either the control or EVOO group $(\mathrm{P}<0.05$; Table 4$)$.

\section{In vitro aortic response to phenylephrine in the absence and presence of metformin}

In generating the concentration-response curves for phenylephrine, there were no significant differences detected between the diet groups $(\mathrm{P}>0.05)$ (Figure 3A). However, incubation of the aortic rings with metformin $\left(10^{-4} \mathrm{M}\right)$ significantly reduced the vasoconstrictor response $(\mathrm{P}<0.05)$ to phenylephrine, but the effect was lower in both high-fat diet groups than in the control group (Figure $3 \mathrm{~B}$ ).

Relaxation response to carbachol or sodium nitroprusside in the absence or presence of metformin

The addition of phenylephrine $\left(1 \times 10^{-6} \mathrm{M}\right)$ to the incubating medium induced a maximum contraction that 

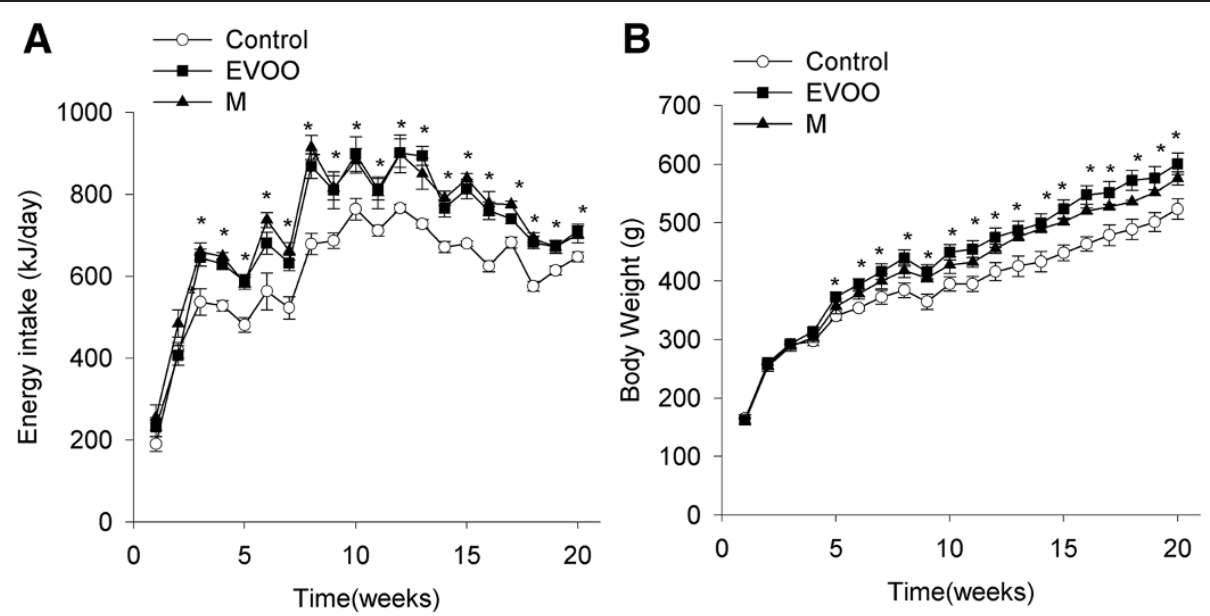

Figure 1 Changes in body weight and energy intake. Energy intake (A) and body weight (B) of rats fed with control or high-fat olive oil or margarine diets for 20 weeks. ${ }^{*} P<0.05$, significant difference compared with the control group by 2-way RM ANOVA ( $n=9$ per group).

reached a plateau. Subsequently, the concentrationresponse curve for sodium nitroprusside was determined for each group $\left(1 \times 10^{-8}\right.$ to $\left.1 \times 10^{-4} \mathrm{M}\right)$, both in the presence and absence of metformin. The relaxing effect of carbachol was significantly lower in the M-fed rats compared with the EVOO-fed or control groups (Figure $3 \mathrm{C}$ ). Metformin increased the relaxation produced by carbachol in all of the dietary groups, but the metformin effect was less pronounced in the $\mathrm{M}$-fed group (Figure 3D). There were no significant differences in the relaxation response to sodium nitroprusside of the experimental groups, either in the presence or absence of metformin $(\mathrm{P}>0.05)$, but there was a significant difference within each group in their responses to sodium nitroprusside before and after the addition of metformin to the incubation medium (Figure 3E and 3F).

\section{Discussion}

The high consumption of energy-dense vegetable fat and physical inactivity are considered major causes of obesity. Many studies have demonstrated that high-fat diets have contributed to the increased prevalence of insulin resistance [44-46] and to the eventual development of diabetes in genetically susceptible individuals and populations $[31,47,48]$. This study shows that two different vegetable fat have different metabolic and vascular effects in rats subjected to high-fat diets of isoenergetic value. Ingesting high levels of extra virgin olive oil produces obesity and insulin resistance in study rats, which is comparable to what is observed in margarine-fed rats, but only the margarine-heavy diet produced hypercholesterolemia and significant endothelial dysfunction. In agreement with other reports $[4,49,50]$ the rats fed HF diets ingested more energy and gained more weight than
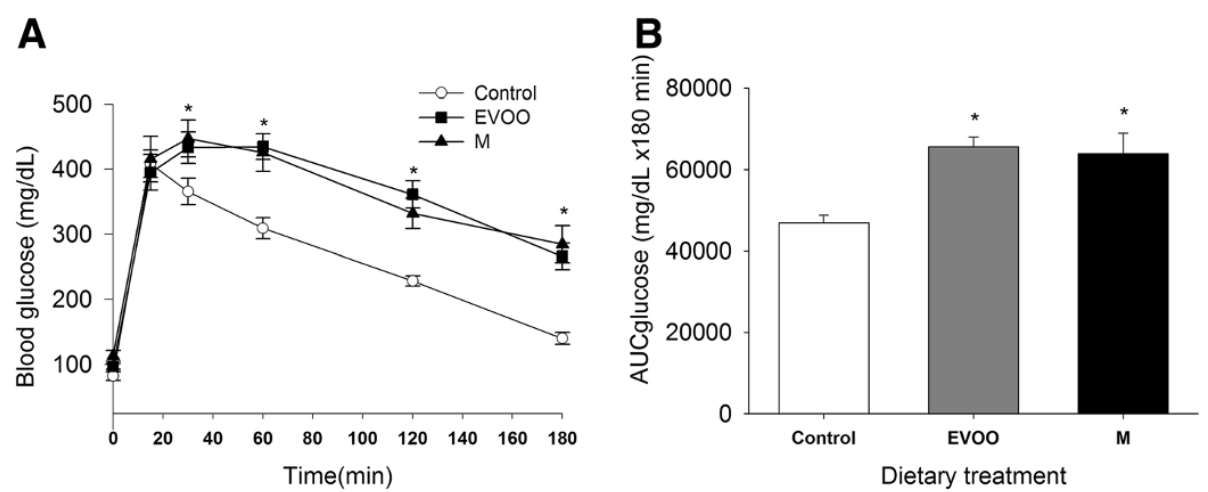

Figure 2 Effect of the HF-diets on blood glucose levels. Glycemia (A) and the area under the glucose curve (B) after 12 hours of fasting following an IP injection of $3 \mathrm{~g} / \mathrm{kg}$ of glucose solution in rats fed a control or a high-fat diet (EVOO or M) for 20 weeks by 2 -way RM ANOVA (for $\mathbf{A})$ and one-way ANOVA (for B). ${ }^{*} \mathrm{P}<0.05$, significant difference compared with the control group. ( $\mathrm{n}=9$ per group). 
Table 2 Fasting blood glucose, plasma insulin levels and HOMA-IR after 20 weeks of dietary treatment

\begin{tabular}{lccc}
\hline & Control & EVOO & M \\
\hline Glucose $(\mathbf{m g} / \mathbf{d L})$ & $92.5 \pm 3.2$ & $97.1 \pm 4.2$ & $113.1 \pm 8.3^{*} \&$ \\
Insulin $(\boldsymbol{\mu U} / \mathbf{m L})$ & $22.51 \pm 0.11$ & $22.76 \pm 0.52$ & $22.93 \pm 0.28$ \\
HOMA-IR & $0.81 \pm 0.03$ & $1.07 \pm 0.08^{*}$ & $1.14 \pm 0.11^{*}$
\end{tabular}

Values are expressed as the means \pm SEM ( $n=9$ per group). ${ }^{*} P<0.05$, significant difference compared with the control group; \& $P<0.05$, significant difference compared with the EVOO group by one-way ANOVA.

the control rats did. Contrary to our results, some studies support an anti-obesogenic effect of EVOO [33,51], but other longer-term studies report weight gain with sustained EVOO intake [35]. This discrepancy could be partly explained by the duration of dietary treatment (4 weeks vs. 12 weeks) or the quantity of EVOO administered [34]. Indeed, our results support that obesity depends on the quantity of fat ingested, its composition and the duration of dietary treatment. This is because high-fat feeds in rats require at least 4 weeks of treatment to produce a significant increase in body weight, and both monounsaturated and saturated fatty acids favor adiposity. In recent years, the adherence to the Mediterranean diet has been associated with lower risk for cardiovascular disease [6]. This diet is characterized by the high consumption of fruits, vegetables, cereals, fish and virgin olive oil. This Mediterranean dietary pattern has a low energy density and a high fiber content that contributes to satiation which may explain the lower body weight, abdominal fat deposition and fasting blood glucose reported with the adherence to this diet $[7,52]$. In contrast, our results show that feeding rats with the hypercaloric and unbalanced high-fat diets based on EVOO or M, produce an increase in energy ingestion that may lead to the accumulation of visceral fat, which in turn is linked to the progression of insulin resistance and impaired glucose tolerance $[35,53,54]$. In our rats, $3 \mathrm{hrs}$ after the administration of a glucose load, blood glucose levels had decreased to their basal levels in the control group, only. The lower capacity of rats maintained on HF diets (EVOO or M) to return to their basal levels of glucose is indicated by the greater areas

Table 3 Serum total cholesterol, HDL, LDL and TG concentrations, and intraabdominal fat

\begin{tabular}{llll}
\hline & Control & EVOO & M \\
\hline Total Cholesterol $(\mathbf{m g} / \mathbf{d L})$ & $71.54 \pm 4.6$ & $72.31 \pm 2.6$ & $84.0 \pm 5.0^{*}$ \\
TG $(\mathbf{m g} / \mathbf{d L})$ & $58.46 \pm 5.3$ & $67.32 \pm 3.5$ & $65.54 \pm 4.4$ \\
HDL cholesterol $(\mathbf{m g} / \mathbf{d L})$ & $19.72 \pm 2.3$ & $21.6 \pm 2.3$ & $18.9 \pm 2.3$ \\
LDL cholesterol $(\mathbf{m g} / \mathbf{d L})$ & $40.0 \pm 5.2$ & $37.3 \pm 3.4$ & $52.0 \pm 5.0^{*}$ \\
Intraabdominal fat & $5.18 \pm 0.57$ & $9.06 \pm 0.44^{*}$ & $8.71 \pm 0.63^{*}$ \\
(g/100 g body weight) & & & \\
\hline
\end{tabular}

* $P<0.05$, significant difference compared with the control group by one-way ANOVA ( $n=9$ per group). Values are expressed as the means \pm SEM. under their glucose curves (Figure 2). Moreover, the HOMA-IR analysis, which indicates insulin resistance under fasting conditions, revealed significant differences between the HF-diet groups and the control group, with the HF-fed rats showed signs of insulin resistance. Consistent with this result, it has already been shown that HF diets contribute to glucose intolerance and the subsequent development of insulin hypersecretion after a glucose load $[50,55]$ because the body attempts to keep glucose at normal physiological levels. Several studies have reported that visceral adipocytes are less responsive than subcutaneous fat cells to the antilipolytic effect of insulin [56,57] and that exposure of the liver to high concentrations of free fatty acids can induce changes in insulin signaling that promote hepatic insulin resistance [58]. In summary, the metabolic impact of high oleic acid intake on glucose and lipid metabolism is not conclusive in humans, whereas it clearly leads to obesity and insulin resistance in rats [35]. Although, dietary fat composition plays an important role in the prevention of cardiovascular disease (CVD) and metabolic syndrome $[59,60]$, it has been suggested that the total intake of fat (SAFA, MUFA and PUFA), regardless of the quality of the fat, can increase the risk of CVD [23]. In this study, EVOO and $\mathrm{M}$ diets have shown to exert different effects on the vasomotor response of endothelial tissue, showing signs of altered function in the margarine-fed group, only. Extra virgin olive oil is rich in unsaturated fatty acids, mainly oleic acid, and phenolic compounds [12], which contribute to its cardioprotective effects [14]. In contrast to EVOO, margarine has a high saturated fatty acid content, which is produced industrially via the hydrogenation of unsaturated oils [61]. Many studies have shown that consumption of these fats contributes to an increased cardiovascular risk [23]. As our results show, lipid peroxidation was elevated only in the rats that were maintained on a high- $\mathrm{M}$ diet. These results suggest that the consumption of a high-M diet, over a period of 20 weeks, leads to increased levels of oxidative stress, hypercholesterolemia and insulin resistance which in turn lead to alterations in tissue function, including endothelial vasomotor response. These findings are consistent with many studies that have shown that the antioxidant properties of EVOO are mainly due to its high content of polyphenolic compounds [12], which are strong antioxidants and radical scavengers [62,63]. In contrast, saturated fatty acids increase oxidative stress and thus modify the lipid composition of cell membranes $[64,65]$. Consistent with these studies, our results indicate that the serum lipid profiles of rats maintained on the $\mathrm{M}$ diet reflected higher levels of total and LDL cholesterol compared with the rats fed high-EVOO or control diets. It is well documented that hypercholesterolemia is associated with a higher incidence of 
Table 4 Malondialdehyde levels in the aorta, heart and liver

\begin{tabular}{lccr}
\hline Tissues & Control & EVOO & M \\
\hline Arterial MDA (mM/mg Protein) & $15.76 \pm 1.83$ & $22.94 \pm 4.58$ & $50.46 \pm 5.56^{*} \&$ \\
Heart MDA (mM/mg Protein) & $3.77 \pm 0.34$ & $3.83 \pm 0.59$ & $7.13 \pm 0.20^{* *} \&$ \\
Hepatic MDA (mM/mg Protein) & $3.09 \pm 0.22$ & $3.25 \pm 0.34$ & $5.62 \pm 0.47 * \&$ \\
\hline
\end{tabular}

Values are expressed as the means \pm SEM ( $n=9$ per group). MDA malondialdehyde. ${ }^{*} \mathrm{P}<0.05$, significant difference compared with the control group. \& $\mathrm{P}<0.05$, significant difference between the EVOO and M groups according to one-way ANOVA.
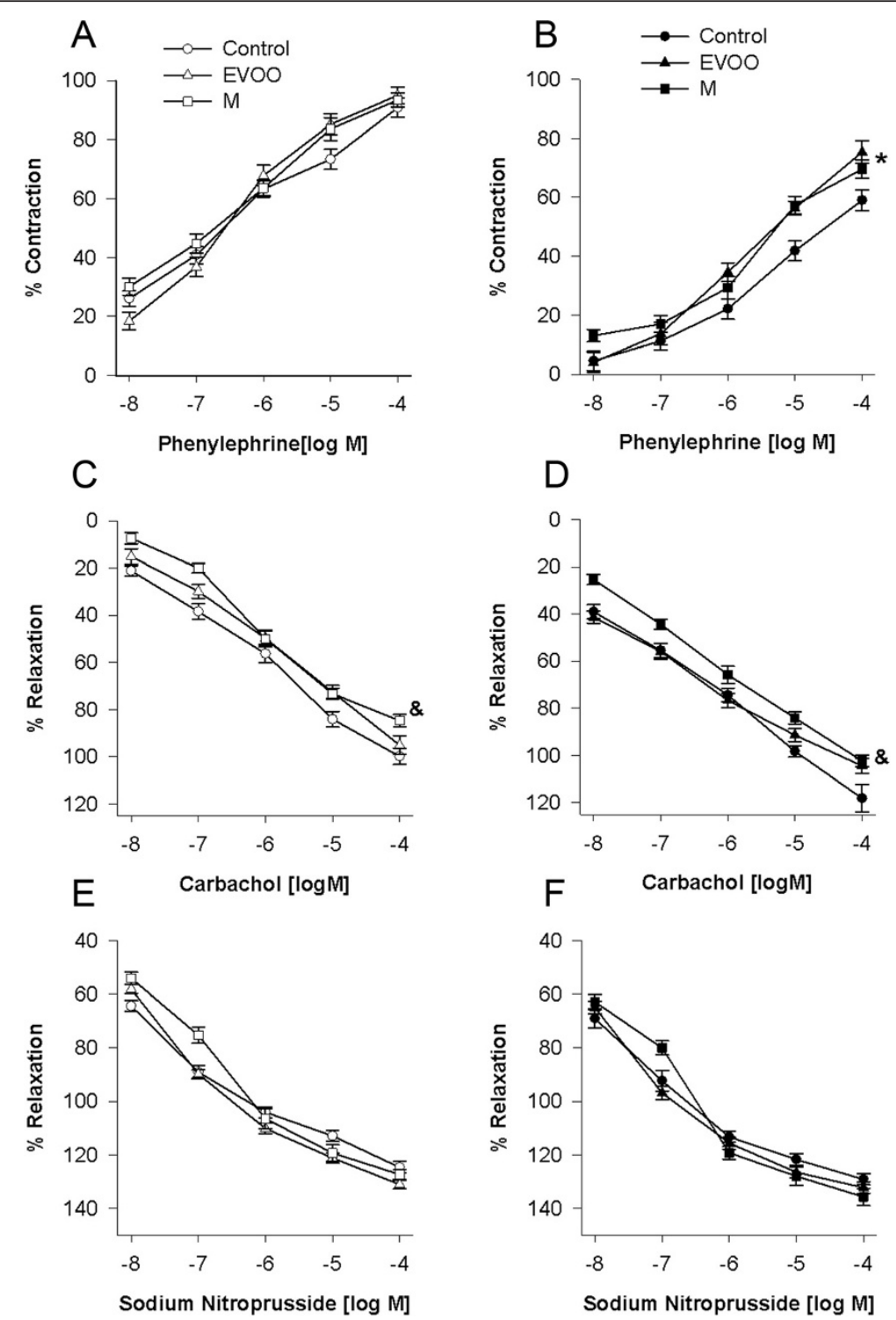

Figure 3 Effects of the HF-diets on endothelial function. Concentration-response curves for phenylephrine (A) and (B), carbachol (C and D) or sodium nitroprusside ( $\mathbf{E}$ and $\mathbf{F}$ ) before and after metformin addition to the incubation media of thoracic aortic rings obtained from age-matched control, EVOO and $\mathrm{M}$ rats ( $\mathrm{n}=9$ per group). The results show the contraction of the thoracic aortic rings as a percentage of the maximal contraction induced by phenylephrine $\left(10^{-8}\right.$ to $\left.10^{-4} \mathrm{M}\right)$ and the relaxation as a percentage of the effect induced by carbachol $\left(1 \times 10^{-8}\right.$ to $\left.1 \times 10^{-4} \mathrm{M}\right)$ or sodium nitroprusside $\left(1 \times 10^{-8}\right.$ to $\left.1 \times 10^{-4} \mathrm{M}\right)$. ${ }^{*} \mathrm{P}<0.05$ significant difference compared with the control group; \& $\mathrm{P}<0.05$ significant difference compared with the control and the EVOO groups by 2-way RM ANOVA. Values are the means \pm SEM. 
atherosclerosis [66]. In addition, although there was no significant difference in the levels of TG, there was an increasing trend in this parameter in M-fed rats, which suggests that, even though all of the rats that were fed high-fat diets (EVOO or M) were obese, the EVOO-high diet still conferred a lower cardiovascular risk compared with the M-high diet. Given that obesity has been linked to insulin resistance and increased inflammation, causing endothelial dysfunction, EVOO-fed rats should also be expected to show signs of altered endothelial function. Carbachol-induced NO production and vasodilation are conserved in these rats, however, suggesting that the expected endothelial damage had been prevented. It has been shown that oleic acid, the major fatty acid constituent in EVOO and polyphenols, diminishes the oxidation of olive oil-derived lipoproteins, thus mitigating any potential oxidative damage to endothelial vasomotor function. These results support the finding that, among EVOO compounds, polyphenols, mainly hydroxytyrosol and tyrosol, increase NO production and prevent the formation of the powerful oxidant peroxynitrite [26]. The concentration-response curve for phenylephrine showed that the high-fat diets did not modify the endothelium's vasoconstrictive response to this substance, nor did they affect the tissue's vasodilatory response to sodium nitroprusside. However, the $M$ diet did reduce the relaxation generated by carbachol, suggesting that the ingestion of significant amounts of saturated fat affected the endothelium-dependent vasorelaxation response in the rats fed the $\mathrm{M}$ diet. Hypercholesterolemia and high levels of lipid peroxidation in the arteries are likely to be associated with the alteration in vascular response because it is well known that inflammation plays a key role in the onset of atherosclerosis and oxidative stress and may also contribute to increased endothelial damage [67]. Earlier studies support the hypothesis that endothelial dysfunction is associated with a reduction in the release [68] or availability of NO, the production of which is downregulated in the presence of reactive oxygen species (ROS), the production of which, in turn, is generally increased in the obese state [31]. Although the results of this study showed an increased aortic lipoperoxidation in $\mathrm{M}$ fed rats, and there were significant differences in endothelial response to vasorelaxing substances among HF diets groups, the differences in endothelial reactivity were lower than was expected for the high values of lipoperoxidation in M-fed rats, suggesting that the quantity of fats in the diets $(25 \% \mathrm{w} / \mathrm{w})$ was very high and duration of the regimen diet (20 weeks) was too long, producing some common alterations not related to lipoperoxidation in both HF rat diets. Furthermore, there were no high differences when comparing to control rats, thus indicating as previously suggested that the process of maturation and aging in rat lead to degenerative changes at the cellular level including arterial endothelial cells function [69]. Indeed, a reduction in the dietary intervention could show higher differences in endothelial function between EVOO and M diets. As our results show, there was no significant difference in the vasodilatory responses to sodium nitroprusside in the arteries from the experimental groups, which suggests that the limited vasodilatory response to carbachol (muscarinic agonist) that was observed in the arteries of the M-fed rats was due to a lower endothelial production of NO. The diets did not affect the endothelial response to alpha1-adrenergic (phenylephrine) or beta-adrenergic (isoproterenol, results not shown) agonists, which may indicate that the diets had no effect on the adrenergic vascular response. Furthermore, this study corroborates previous findings that incubation of the aorta rings from streptozotocin-induced diabetic rats with metformin enhanced the carbachol-induced relaxation and reduced the phenylephrine-induced contraction, which have been reported to be endothelium dependent [70], similarly, in our insulin resistant rats, the addition of metformin to the incubation media showed a lower reduction of the phenylephrine-induced contraction in both high fat diet fed rats compared to control rats, but an even lower response to carbachol in $\mathrm{M}$ rats, indicating that just high $\mathrm{M}$ diet altered both endothelium dependent relaxing mechanisms. Metformin vasodilatory effects have been proposed to be associated with the activation of AMPactivated protein kinase which increases the activity of endothelial NO synthase [71]. Additionally, the enhanced vasodilator effect of nitroprusside indicates that metformin also produces relaxation through a mechanism that is independent of endothelial NO production, possibly via the decreased production of vasoconstricting prostanoids [72] or decreased intracellular calcium [73], both of which are factors that could be altered and by doing so, contributing to lower the endothelial response in M-fed rats.

\section{Conclusions}

In conclusion, we found that the long-term high-fat intake lead to obesity and insulin resistance, regardless of the fat composition (saturated or monounsaturated). Nevertheless, EVOO did not increase plasma levels of total cholesterol or LDL cholesterol, nor did it increase arterial lipid peroxidation, which may contribute to the preservation of endothelial response to carbachol, effect that is significantly enhanced by metformin only in rats fed with control and EVOO diets. Further to this preliminary study, longer-term animal and in vitro studies are needed to better understand the mechanisms regarding the ingestion of a diet high in extra virgin olive oil to produce obesity and insulin resistance but protect endothelial function. 


\section{Abbreviations}

CVD: Cardiovascular disease; EVOO: Extra virgin olive oil; GTT: Glucose tolerance test; HDL cholesterol: High-density lipoprotein cholesterol; HF-diet: High-fat diet; HOMA-IR: Homeostatic model assessment of insulin resistance; IP: Intraperitoneal; KHS: Krebs Hanseleit solution; LDL cholesterol: Low-density lipoprotein cholesterol; M: Margarine; MDA: Malondialdehyde; MUFA: Monounsaturated fatty acids; PUFA: Polyunsaturated fatty acids; ROS: Reactive oxygen species; SAFA: Saturated fatty acids; TG: Triglycerides.

\section{Competing interests}

The authors declare that they have no competing interests.

\section{Authors' contributions}

Conceived and designed the experiments: ERSJ, and LQ. Performed the experiments and analyzed the data: HK, ERSJ, LGC and NPC. HK and NPC participated in its design and coordination and helped to draft the manuscript. Wrote the paper: LQ. All authors read and approved the final manuscript.

\section{Acknowledgments}

The authors would like to acknowledge SIP/IPN grant 20131172 for the financial support. H. Keita thanks PIFI, Instituto Politécnico Nacional and CONACYT for granting a graduate scholarship awarded. E. Ramírez-San Juan, N. Paniagua-Castro, L. Garduño-Siciliano and L. Quevedo are fellows of EDI and COFAA/IPN programs.

\section{Author details}

'Departamento de Fisiología, Escuela Nacional de Ciencias Biológicas, Instituto Politécnico Nacional, Carpio y Plan de Ayala, México, D.F., México. ${ }^{2}$ Farmacia de la Escuela Nacional de Ciencias Biológicas, Instituto Politécnico Nacional, México, D.F., Mexico.

Received: 28 January 2013 Accepted: 12 September 2013

Published: 18 September 2013

\section{References}

1. Popkin BM: The world is fat. Sci Am 2007, 29:88-95.

2. Engelgau MM, Geiss LS, Saaddine JB, Boyle JP, Benjamin SM, Gregg EW, Tierney EF, Rios Burrows N, Mokdad AH, Ford ES, Imperatore G, Narayan KM: The evolving diabetes burden in the United States. Ann Intern Med 2004, 140:945-950.

3. Gregg EW, Cheng YJ, Cadwell BL, Imperatore G, Williams DE, Flegal KM, Naravan KM, Williamson DF: Secular trends in cardiovascular disease risk factors according to body mass index in US adults. JAMA 2005, 293:1868-1874.

4. Su W, Jones PJH: Dietary fatty acid composition influences energy accretion in rats. J Nutr 1993, 123:2109-2114.

5. Hill JO, Peters JC, Lin D, Yakabu F, Greene H, Swift L: Lipid accumulation and body fat distribution are influenced by type of dietary fat fed to rats. Int J Obes Relat Metab Disord 1993, 17:223-236.

6. Trichopoulou A, Costacou T, Bamia C, Trichopoulos D: Adherence to a Mediterranean diet and survival in a Greek population. $N$ Engl J Med 2003, 348:2599-2608.

7. Di Daniele N, Petramala L, Di Renzo L, Sarlo F, Della Rocca DG, Rizzo M, Fondacaro V, lacopino L, Pepine CJ, De Lorenzo A: Body composition changes and cardiometabolic benefits of a balanced Italian Mediterranean Diet in obese patients with metabolic syndrome. Acta Diabetol 2013, 50:409-416.

8. Perez-Jimenez F, Lopez-Miranda J, Pinillos MD, Gomez P, et al: A Mediterranean and high carbohydrate diet improve glucose metabolism in healthy young person's. Diabetologia 2001, 44:2038-2043.

9. Esposito K, Marfella R, Ciotola M, Di Palo C, Giugliano F, Giugliano G, et al: Effect of a Mediterranean style diet on endothelial dysfunction and markers of vascular inflammation in the metabolic syndrome: a randomized trial. JAMA 2004, 292:1440-1446.

10. Sanchez-Villegas A, Bes-Rastrollo M, Martinez-Gonzalez MA, Serra-Majem L: Adherence to a mediterranean dietary pattern and weight gain in a follow up study: the SUN cohort. Int J Obes 2006, 30:350-358.
11. Panagiotakos DB, Polychronopoulos E: The role of Mediterranean diet in the epidemiology of metabolic syndrome; converting epidemiology to clinical practice. Lipids Health Dis 2005, 4:7.

12. Martín-Peláez S, Covas MI, Fitó M, Kušar A, Pravst I: Health effects of olive oil polyphenols: recent advances and possibilities for the use of health claims. Mol Nutr Food Res 2013, 57:760-771.

13. Stark AH, Mader Z: Olive oil as a functional food: epidemiology and nutritional approaches. Nutr Rev 2002, 60:170-176.

14. Bogani P, Galli C, Villa M, Visioli F: Postprandial anti-inflammatory and antioxidant effects of extra virgin olive oil. Atherosclerosis 2007. 190:181-186.

15. Moreno-Luna R, Muñoz-Hernandez R, Miranda ML, Costa AF, JimenezJimenez L, Vallejo-Vaz AJ, Muriana FJG, Villar J, Stiefel P: Olive oil polyphenols decrease blood pressure and improve endothelial function in young women with mild hypertension. Am J Hypertens 2012, 25:1299-1304.

16. Fabiani R, Rosignoli P, De Bartolomeo A, Fuccelli R, Servili M, Montedoro GF, Morozzi G: Oxidative DNA damage is prevented by extracts of olive oil, hydroxytyrosol, and other olive phenolic compounds in human blood mononuclear cells and HL60 cells. J Nutr 2008, 138:1411-1416.

17. Musumeci G, Trovato FM, Imbesi R, Castrogiovanni P: Effects of dietary extra-virgin olive oil on oxidative stress resulting from exhaustive exercise in rat skeletal muscle: A morphological study. Acta Histochem 2013. http://dx.doi.org/10.1016/j.acthis.2013.05.006.

18. Mattson FH, Grundy SM: Comparison of effects of dietary saturated, monounsaturated, and polyunsaturated fatty acids on plasma lipids and lipoproteins in man. J Lipid Res 1985, 26:194-202.

19. Ascherio A, Rimm EB, Giovannucci EL, Spiegelman D, Stampfer MJ, Willett WC: Dietary fat and risk of coronary heart disease in men cohort follow up study in the United States. BMJ 1996, 313:84-90.

20. Hu FB, Stampfer MJ, Manson JE, Rimm E, Colditz GA, Rosner BA, Hennekens $\mathrm{CH}$, Willett WC: Dietary fat intake and the risk of coronary heart disease in women. N Engl J Med 1997, 337:1491-1499.

21. Judd JT, Baer DJ, Clevidence BA, Kris-Etherton P, Muesing RA, Iwane M: Dietary cis and trans monounsaturated and saturated FA and plasma lipids and lipoproteins in men. Lipids 2002, 37:123-231.

22. Chen CL, Tetri LH, Neuschwander-Tetri BA, Huang SS, Huang JS: A mechanism by which dietary trans fats cause atherosclerosis. I Nutr Biochem 2011, 22:649-655.

23. Erkkilä A, de Mello VD, Risérus U, Laaksonen DE: Dietary fatty acids and cardiovascular disease: an epidemiological approach. Pro Lipid Res 2008, 47:172-187

24. Steinberg HO, Tarshoby M, Monestel R, Hook G, Cronin J, Johnson A Bavazeed B, Baron AD: Elevated circulating free fatty acid levels impair endothelium-dependent vasodilatation. J Clin Invest 1997, 100:1230-1239.

25. Inoguchi T, Li P, Umeda F, Yu HY, Kakimoto M, Imamura M, Aoki T, Etoh T, Hashinmoto T, Naruse M, Sano H, Utsumi H, Nawata H: High glucose level and free fatty acid stimulate reactive oxygen species production through protein kinase $\mathrm{C}$ dependent activation of $\mathrm{NAD}(\mathrm{P}) \mathrm{H}$ oxidase in cultural vascular cells. Diabetes 2000, 49:1939-1945.

26. Perona JS, Cabello-Moruno R, Ruiz-Gutierrez V: The role of virgin olive oil components in the modulation of endothelial function. J Nutr Biochem 2006, 17:429-445.

27. Berg AH, Scherer PE: Adipose tissue, inflammation, and cardiovascular disease. Circ Res 2005, 96:939-949.

28. Schaffler A, Muller-Ladner U, Scholmerich J, Buchler C: Role of adipose tissue as an inflammatory organ in human diseases. Endocr Rev 2006, 27:449-467.

29. Muniyappa R, Montagnani, Kon Koh K, Quon MJ: Cardiovascular actions of insulin. Endocr Rev 2007, 28:463-491.

30. Moritoki H, Tanioka A, Maeshiba Y, Iwamoto T, Ishida Y, Araki H: Ageassociated decrease in histamine induced vasodilatation may be due to reduction of cyclic GMP formation. Br J Pharmacol 1988, 95:1015-1022.

31. Jonk AM, Houben AJ, de Jongh RT, Serné EH, Schaper NC, Stehouwer CD: Microvascular dysfunction in obesity: a potential mechanism in the pathogenesis of obesity-associated insulin resistance and hypertension. Physiology 2007, 22:252-260.

32. de Vriese AS, Verbeuren TJ, Van de Voorde J, Lameire NH, Vanhoutte PM: Endothelial dysfunction in diabetes. Br J Pharmacol 2000, 130:963-974.

33. Oi-Kano Y, Kawada T, Watanabe T, Koyama F, Watanabe K, Senbongi R, Iwai $K$ : Extra virgin olive oil increases uncoupling protein 1 content in brown 
adipose tissue and enhances noradrenaline and adrenaline secretions in rats. J Nutr Biochem 2007, 18:685-692.

34. Alsaif MA, Duwaihy MMS: Influence of dietary fat quantity and composition on glucose tolerance and insulin sensitivity in rats. Nutr Res 2004, 24:417-425.

35. Buettner R, Parhofer KG, Woenckhaus M, Wrede CE, Kunz-Schughart A, Schölmerich J, Bollheimer LC: Defining high-fat diet rat models metabolic and molecular effects of different fat types. J Mol Endocrinol 2006, 36:485-501.

36. Ramirez-Tortosa MC, Urbano G, López-Jurado M, Nestares T, Gomez MC, Mir A, Ros E, Mataix J, Gil A: Extra-virgin olive oil increases the resistance of LDL to oxidation more than refined olive oil in free-living men with peripheral vascular disease. J Nutr 1999, 129:2177-2183.

37. Friedeward WT, Levy RI, Fredrickson DS: Estimation of the concentration of low- density lipoprotein cholesterol in plasma, without use of the preparative ultracentrifuge. Clin Chem 1972, 18:499-502

38. Gelardi NL, Chung-Ja MC, William OH: Evaluation of insulin sensitivity in obese offspring of diabetic rats by hyperinsulinemic-euglycemic clamp technique. Pediatr Res 1991, 30:40-44.

39. Shen L, Tso P, Woods SC, Sakai RR, Davidson WS, Liu M: Hypothalamic apolipoprotein A IV is regulated by leptin. Endocrinology 2007, 148:2681-2689.

40. Cacho J, Sevillano J, de Castro J, Herrera E, Ramos MP: Validation of simple indexes to assess insulin sensitivity during pregnancy in Wistar and Sprague-Dawley rats. Am J of Physiol-Endocrinol Metab 2008, 295:1269-1276.

41. Buege JA, Aust SD: Microsomal lipidperoxidation. Methods Enzymol 1978, 52:302-310.

42. Bradford MM: A rapid and sensitive method for the quantitation of microgram quantities of protein utilizing the principle of protein-dye binding. Anal Biochem 1976, 72:248-254.

43. Katakam PVG, Ujhelyi MR, Hoenig M, Miller AW: Metformin improves vascular function in insulin-resistance rats. Hypertension 2000, 35:108-112.

44. Chalkley SM, Hettiarachchi M, Chisholm DJ, Kraegen EW: Long-term highfat feeding leads to severe insulin resistance but not diabetes in Wistar rats. Am J Physiol Endocrinol Metab 2002, 282:1231-1238.

45. Han DH, Hansen PA, Host HH, Holloszy JO: Insulin resistance of muscle glucose transport in rats fed a high-fat diet: a reevaluation. Diabetes 1997, 46:1761-1767.

46. Axen $\mathrm{KV}$, Axen K: Longitudinal adaptations to very low-carbohydrate weight-reduction diet in obese rats: Body composition and glucose tolerance. Obesity 2010, 18:1538-1544.

47. Corsetti JP, Sparks JD, Peterson RG, Smith RL, Sparks CE: Effect of dietary fat on the development of non-insulin dependent diabetes mellitus in obese Zucker diabetic fatty male and female rats. Atherosclerosis 1999 , 148:231-241.

48. Barnett $M$, Collier GR, Odea K: Energy intake with respect to the development of diabetes mellitus in psammomys obesus. Diab Nutr Metab 1995, 8:42-47.

49. Rolland V, Roseau S, Fromentin G, Nicolaidis S, Tomé D, Even PC: Body weight, body composition, and energy metabolism in lean and obese Zucker rats fed soybean oil or butter. Am J Clin Nutr 2002, 75:21-30.

50. Cruciani-Guglielmacci C, Vincent-Lamon M, Rouch C, Orosco M, Ktorza A Magnan C: Early changes in insulin secretion and action induced by high-fat diet are related to a decreased sympathetic tone. Am J Physiol Endocrinol Metab 2005, 288:148-154.

51. Landsberg L, Young JB: Diet-induced changes in sympathoadrenal activity: Aplications for thermogenesis. Life Sci 1981, 28:1801-1819.

52. Schröder $\mathrm{H}$ : Protective mechanisms of the Mediterranean diet in obesity and type 2 diabetes. J Nutr Biochem 2007, 18:149-160.

53. Hansen BC: The metabolic syndrome X. Ann N Y Acad Sci 1999, 892:1-24.

54. Kim JY, Nolte LA, Hansen PA, Han DH, Ferguson K, Thompson PA, Holloszy JO: High-fat diet-induced muscle insulin resistance: relationship to visceral fat mass. Am J Physiol-Regul Integr Comp Physiol 2000, 279:2057-2065.

55. Barrientos C, Racotta R, Quevedo L: Glucosamine attenuates increases of intraabdominal fat, serum leptin levels, and insulin resistance induced by a high-fat diet in rats. Nutr Res 2010, 30:791-800

56. Bolinder J, Kager L, Ostman J, Arner P: Differences at the receptor and postreceptor levels between human omental and subcutaneous adipose tissue in the action of insulin on lipolysis. Diabetes 1983, 32:117-123.
57. Zierath J, Livingston J, Thorne A, Bolinder J, Reynisdottir S, Lonnqvist F, Arner P: Regional difference in insulin inhibition of non-esterified fatty acid release from human adipocytes: relation to insulin receptor phosphorylation and intracellular signaling through the insulin receptor substrate-1 pathway. Diabetologia 1998, 41:1343-1354

58. Montague CT, Rahilly SO: The perils of portliness: causes and consequences of visceral adiposity. Diabetes 2000, 49:883-888.

59. Summers LK, Fielding BA, Bradshaw HA, llic V, Beysen C, Clark ML, Moore NR, Frayn KN: Substituting dietary saturated fat with polyunsaturated fat changes abdominal fat distribution and improves insulin sensitivity. Diabetologia 2002, 45:369-377.

60. Warensjo E, Riserus U, Vessby B: Fatty acid composition of serum lipids predicts the development of the metabolic syndrome in men. Diabetologia 2005, 48:1999-2005.

61. Emken EA: Nutrition and biochemistry of trans and positional fatty acid isomers in hydrogenated oils. Annu Rev Nutr 1984, 4:339-379.

62. Lavelli $\mathrm{V}$ : Comparison of the antioxidant activities of extra virgin olive oils. J Agri Food Chem 2002, 50:7704-7708.

63. Vissera MN, Zock PL, Katan MB: Bioavailability and antioxidant effects of olive oil phenols in humans. Eur J Clin Nutr 2004, 58:955-965.

64. Peres CM, Procopio J, Costa M, Curi R: Thioglycolate elicited rat macrophages exhibit alterations in incorporation and oxidation of fatty acids. Lipids 1999, 34:1193-1197.

65. Lee JY, Sohn KH, Rhee SH, Wang DH: Saturated fatty acids, but not unsaturated fatty acids, induce the expression of cyclooxygenase-2 mediated through Toll-like receptor 4. J Bio/ Chem 2001, 276:16683-16689.

66. Ursini F, Sevanian A: Postprandial oxidative stress. Biol Chem 2002, 383:599-605.

67. Stocker R, Keaney JF: Role of oxidative modifications in atherosclerosis. Physiol Rev 2004, 84:1381-1478.

68. Endo K, Abiru T, Machida H, Kasuya Y, Kamata K: Endothelium derived hyperpolarizing factor does not contribute to the decrease in endothelium-dependent relaxation in the aorta of streptozotocin induced diabetic rats. Gen Pharmacol 1995, 26:146-153.

69. Félétou M, Vanhoutte PM: Endothelial dysfunction: a multifaceted disorder. Am J Physiol Heart Circ Physiol 2006, 291:985-1002.

70. Majithiya JB, Balaraman R: Metformin reduces blood pressure and restores endothelial function in aorta of streptozotocin-induced diabetic rats. Life Sci 2006, 78:2615-2624.

71. Davis BJ, Xie Z, Viollet B, Zou MH: Activation of the AMP-activated kinase by antidiabetes drug metformin stimulates nitric oxide synthesis in vivo by promoting the association of heat shock protein 90 and endothelial nitric oxide synthase. Diabetes 2006, 55:496-505.

72. Matsumoto T, Noguchi N, Ishida K, Kobayashi T, Yamada N, Kamata K: Metformin normalizes endothelial function by suppressing vasoconstrictor prostanoids in mesenteric arteries from OLETF rats, a model of type 2 diabetes. Am J Physiol Heart Circ Physiol 2008, 295:1165-1176.

73. Chen $\mathrm{XL}$, Panek K, Rembold CM: Metformin relaxes rat tail artery by repolarization and resultant decreases in calcium influx and intracellular calcium. J Hypertension 1997, 15:269-274.

doi:10.1186/1758-5996-5-53

Cite this article as: Keita et al:: The long-term ingestion of a diet high in extra virgin olive oil produces obesity and insulin resistance but protects endothelial function in rats: a preliminary study. Diabetology \& Metabolic Syndrome 2013 5:53. 